\title{
ANÁLISE DA FIDEDIGNIDADE DA DECLARAÇÃO DA CAUSA BÁSICA DE MORTE POR CÂNCER EM SALVADOR, BRASIL
}

Anita Schnitman*

SCHNITMAN, A. Análise da fidedignidade da declaração de causa básica de morte por câncer em Salvador, Brasil. Rev. Saúde públ., S. Paulo, 24:490-6, 1990.

RESUMO: Foi determinada a precisão das informações contidas nas estatísticas de mortalidade por câncer, comparando-se a causa básica de morte da declaração de óbito (DO) com dados de laudo đe anatomia patológica e de diagnóstico hospitalar de uma amostra de 966 declarações de óbito do total de mortes registradas na cidade de Salvador, no ano de 1983. Os laudos de anatomia patológica e dados de prontuários médicos hospitalares confirmam as informaçōes das 485 declaraçōes de óbito que se referiam aos neoplasmas selecionados,em apenas $65 \%$ das vezes. Além de observarmos este baixo percentual de confirmados para os neoplasmas selecionados, encontramos 35 declaraçōes de óbito de indivíduos que tiveram câncer, com comprovação histológica, numa amostra aleatória de 481 declarações de óbito que se referiam a outras causas de morte. Projetando esses achados para as 10.098 mortes restantes teríamos aproximadamente 700 declaraçōes de óbito cuja causa básica deveria ser câncer e não a registrada na declaração de óbito.

DESCRITORES: Atestados de óbitos. Causa de morte. Neoplasias, mortal.

\section{INTTRODUÇÃO}

Dados de mortalidade derivados de declaração de óbito - DO - permitem uma análise numérica ao tempo em que expressam marcante evento do processo de evolução do ser humano. Pelo simples fato de ser obrigatório para a grande maioria de países desenvolvidos, ou seja, ninguém pode ser sepultado sem uma DO, os dados nela existentes permitem que se reconstitua a memória de um povo, de uma civilização; são os fatos científicos que podem ser utilizados para estudar a existência humana.

As variações de sexo, idade, tipo de ocupação, local de residência, nacionalidades e outras diferenças entre os indivíduos que vão a óbito, freqüentemente sugerem explicaçōes alternativas para as causas de morte desses mesmos indivíduos. Daí, o grande número de pesquisas tendo como objeto de interesse a validade das estatísticas de mortalidade $3,4,5,7,10,12$.

A questão da migração, por exemplo, é um fator limitante, que pode influenciar substancialmente os valores das taxas de mortalidade; o problema da validade das informações contidas nas DOs, que se refere à causa básica de morte constituiu-se outra limitação; ainda temos a questão do "período de latência" que é diferente para cada patologia e que impõe limitações diversas ’a interpretação de resultados de pesquisas que utilizam esta fonte de informação.

A qualidade dos dados disponíveis na DO quanto ao câncer especificamente, vem sendo analisada e os achados de alguns desses estudos serão focalizados a seguir.

Autores de um exame detalhado de uma amostra de 8.000 DOs concluíram que todas as localizações anatômicas do câncer estavam sendo diagnosticadas em excesso, numa média de $11 \%$ depois de excluir os cânceres de pele e de lábio ${ }^{1}$.

Uma outra investigação, utilizando uma amostra 2.000 DOs, observou que somente $68 \%$ das 443 mortes atribuídas ao câncer foram comprovadas pelos exames microscópicos dos tecidos. A qualidade das informações que levaram aos diagnósticos foi menos satisfatória para os cânceres de estômago e do pâncreas, e particularmente boa para os órgãos do sistema urinário e para os tecidos hematopoiéticos ${ }^{6}$.

Um outro estudo ${ }^{11}$, realizado nos EUA, comparou dados da $\mathrm{DO}$ com o diagnóstico hospitalar em 48.826 mortes por câncer. Constatou-se tendência em registrar, nas DOs, câncer de localização não especificada enquanto que os diagnósticos hospitalares identificam com maior freqüência a localização anatômica do câncer. Argumentou-se, porém,

Faculdade de Medicina da Universidade Federal da Bahia - Rua Heitor Miguel Calmon, s/n - Vale do Canela - 40240 - Salvador, BA - Brasil. 
que o valor absoluto da taxa de mortalidade não sofre variações significativas para nenhuma localização anatômica em particular, desde que o número de mortes subtraídas ou adicionadas ao numerador é sempre pequeno em relação ao total da população normalmente utilizada ${ }^{11}$.

Uma análise de tendência temporal da mortalidade por neoplasmas malignos relevou que diferenças em diagnóstico e no processo de codificação pode causar uma oscilação muito acentuada para baixo ou para cima para alguns tipos de câncer, especialmente entre os grupos de idade mais avançada. Apesar deste fato, comenta-se na literatura ${ }^{2}$ que os dados provenientes da DO, para a maioria dos cânceres, fornecem indicação razoável da verdadeira tendência correspondente ao real aparecimento da doença.

Vimos assim, que apesar da baixa precisão, a utilização de dados de mortalidade é a única opção que resta a muitos pesquisadores em diversas circunstâncias. Nestes casos, o pesquisador deve e pode: agregar doenças; aumentar o tempo de estudo; limitar a investigação a cidades onde os serviços de saúde tradicionalmente coletam informaçōes de rotina; escolher locais com baixo grau de sub-registro de mortes, onde a maioria dos individuos irão receber assistência médica antes de morrer; onde sómente médicos podem preencher uma DO. Estas estratégias são comumente utilizadas de forma a minimizar os problemas associados à informação vinda de DOs.

O objetivo da presente pesquisa é, pois, determinar a precisão da declaração de óbito enquanto fonte de informação no que se refere a câncer como como causa básica de morte.

\section{MATERIAL E MÉTODO}

A causa básica de morte listada na DO é devidamente codificada por equipe técnica qualificada do Centro de Informaçōes de Saúde (CIS) da Secretaria de Saúde do Estado da Bahia (SESAB) segundo normas da Organização Mundial de Saúde encontradas na publicação "Classificação Internacional das Doenças"8. Além dos códigos referentes à causa básica de morte, as demais informaçōes da DO são rotineiramente transferidas para folhas de codificação com suas normas de preenchimento, para, em seguida, serem enviadas ao Ministério da Saúde (MS), Divisão Nacional de Epidemiologia (DNE), quando, finalmente, são arquivadas em fitas de computador.

As DOs, rotineiramente arquivadas pela $S E S A B$, foram analisadas, e as que tinham como causa básica de morte o câncer foram separadas do "pool" de mortes daquele ano. Do total de mortes por câncer, as que tinham como causa básica de morte os cânceres de estômago, pulmão, fígado, pâncreas, bexiga, linfoma, leucemia e os cânceres de localização não especificada foram consideradas objeto de interesse específico e selecionadas para este estudo, porque:

1. Estes cânceres oferecem uma estimativa adequada da incidência da doença pelas altas razões de letalidade a eles associados e, por isso mesmo, dificilmente passam despercebidos tanto pelo sistema de saúde como um todo, como pelos médicos em particular.

2. Estes neoplasmas têm sido citados na literatura especializada como associados a uma variedade de exposições ocupacionais. A validação da fonte de informação aqui utilizada (DO) fez-se indispensável já que esta indicaria, com alguma margem de segurança, quão próximos da realidade estariam os dados referentes a esses neoplasmas quando a relação entre exposição ocupacional a derivados de petróleo e ocorrência desses tipos de câncer fosse analisada pelo estudo caso-controle, desenvolvido logo em seguida.

3. A informação nas DOs indicava que os indivíduos eram residentes em Salvador, minimizando conseqüentemente o erro normalmente presente de sub-registro de mortes, questão crucial e séria, enfrentada pelo sistema de informação implantado pelo CIS da SESAB, com relação aos demais municípios do nosso Estado.

4. Foram também incluídos no grupo de neoplasmas selecionados os neoplasmas malignos de localização não especificada, pois prevíamos que o erro de preenchimento da causa básica de morte seria consideravelmente maior nesta categoria em relação às demais localizações anatômicas de câncer.

\section{Tamanho da Amostra}

Os neoplasmas selecionados somavam um total de 508 DOs. Dessas, 485 foram aproveitadas. Em 1983, eles representavam 50\% do total de 960 mortes por câncer.

Uma amostra aleatória de 483 mortes foi retirada das 10.556 restantes, de forma a ter um total de DOs que se referiam a qualquer outra causa básica de morte que não os cânceres de interesse, equiparável ao número total de neoplasmas selecionados. Dessas 483 DOs, 481 existiam efetivamente na $S E S A B$ e puderam ser aprọveitados.

Resumindo, tendo como referência os dados do Ministério da Saúde para o ano de 1983, tivemos um total de 991 casos de morte - 508 por neoplasmas selecionados mais 483 mortes por outras causas - a serem investigados a princípio. Em nível estadual, ou seja, no CIS da SESAB encontramos 982 DOs ao invés de 991 . Nove DOs referidas na fita fornecida pela DNE do MS não foram encontradas e 16 listavam endereços de fora de Salvador entre os neoplasmas selecionados. Assim somente 966 
DOs foram examinadas para fins deste estudo. Este número, representou $8,73 \%$ do total de 11.064 mortes para a cidade de Salvador, em 1983.

\section{Coleta de Dados}

Prosseguindo na coleta, além da causa básica de morte e seus respectivos códigos, todas as outras informaçōes relevantes foram transcritas para fichas padronizadas. $\mathrm{Em}$ seguida, foram divididas por local de morte e reorganizadas por ordem alfabética. Cópias destas fichas foram contrastadas quase que simultâneamente com os dados de prontuários médicos dos hospitais (DH) e de arquivos de laboratórios de anatomia patológica (AP) da cidade. Entre as 966 DOs estudadas, havia algumas que se referiam a mortes que ocorreram em casa. Quando nenhuma informação pode ser encontrada nos arquivos de hospitais ou de laboratorios, uma visita ao endereço indicado na DO fez-se necessária. Ainda assim, 59 endereços de indivíduos que a DO indicava neoplasma selecionado não foram localizados. Para efeito de validação da fonte de informação em questão, estas mortes deixaram de ser consideradas como mortes por câncer.

O acordo ou desacordo dessas fontes de informação colocou as 966 DOs no seguinte sistema de classificação:

1. Uma DO que declarava algum dos cânceres em estudo era considerada um verdadeiro positivo(VP) sempre que um laudo de AP, necropsia, laparoscopia, mielograma, ultrasonografia, tomografia, cintilografia e outros possíveis testes de diagnóstico, confirmasse o câncer indicado. O VP para outras causas de morte era identificado quando o diagnóstico da DO coincidia com o diagnóstico de uma das duas fontes de informação aqui utilizadas.

2. A DO que declarava um dos cânceres selecionados e/ou outra causa de morte, que não pôde ser confirmado por uma dessas fontes de informação, foi considerada falso positivo (FP). Numa subcategoria desta categoria foram incluídas as DOs para as quais nenhuma informação pôde ser coletada (FP-SI).

3. Foram classificadas como falso negativo (FN) todas as DOs que declaravam uma localização anatômica de câncer $\mathrm{e}$, mais tarde confirmado ser um câncer de localização selecionada, mas não aquela referida na DO - erro entre os neoplasmas selecionados; assim como, uma morte por cấncer de localização não selecionada - erro entre todos os neoplasmas ou FNs para câncer em geral; como também aquelas DOs que nem citavam câncer e que os dados de AP comprovavam a existência de um câncer - erro ou subestimação do câncer entre outras patologias -, vale ressaltar, estes são os FNs de maior significado neste estudo.

4. Um verdadeiro negativo (VN) foi assim defini- do quando uma DO se referia a uma causa diferente dos neoplasmas selecionados, com evidências diagnósticas a favor da causa referida na DO.

Uma vez assim classificadas, a concordância dessas fontes de informação foi medida através do percentual de confirmados que vem a ser o número de DOs validadas pelas fontes consultadas, sobre o número de DOs analisadas. Este percentual indica a possibilidade de uma DO positiva para um tipo de câncer representar de fato uma morte por este tipo de câncer, tanto quanto, por inversão, nos remete necessariamente à noção de quão superestimada pode estar a estatística oficial.

O número aproximado de subestimados na presente pesquisa, por outro lado, está estritamente relacionado ao número de mortes por câncer com comprovação histológica, encontradas entre as DOs que registravam outra causa de morte que não câncer. Ou seja, o número de verdadeiros $\mathrm{FNs}$ específico por localização, indica quantas mortes por tipo de câncer deixaram de ser computadas em relação ao total de mortes.

\section{RESULTADOS}

As DOs que tinham como causa básica de morte leucemia, linfoma e ca de pulmão foram confirmados em $89 \%, 81 \%$ e $80 \%$ respectivamente, enquanto as DOs que declaravam ca de pâncreas e bexiga foram confirmados em apenas $45 \%$ e $53 \%$, respectivamente (Tabela 1 ). O percentual de confirmação mais baixo, $42 \%$, refere-se às $D O s$ que tinham como causa básica neoplasmas malignos de localização não especificada. Do total de cấnceres selecionados, $65 \%$ foram confirmados à semelhança do percentual encontrados nos EUA. 6,11

Observando os percentuais de DOs FP, temos que $22 \%$ das 485 DOs para neoplasmas selecionados e 25 das 481 para outras causas, não forneciam evidências suficientes que sustentassem o diagnóstico da causa básica de morte referida na DO. Dos neoplasmas estudados, o de pâncreas é o exemplo mais marcante deste tipo de erro. Em aproximadamente $38 \%$ das vezes, DOs tendo ca de pâncreas como causa básica de morte não puderam ser confirmados. Este resultado pode ser explicado pela falta de testes diagnósticos que tenha especificidade alta o suficiente para o diagnóstico de certeza, menos traumático que o existente atualmente. Os testes diagnósticos para ca de pulmão são falhos, enquanto os para fígado e estômago apresentam riscos de complicaçōes, mais para fígado e menos para estômago, o que explica os percentuais de 11,24 e $14 \%$, respectivamente. Para o câncer de bexiga, que teve $20 \%$ de confirmação, esta explicação já não procede. Existem hoje testes para se chegar ao diagnóstico de ca de bexiga, extremamente simples e eficientes.

Refletindo de forma flagrante sobre o problema 
de acesso aos serviços e a estrutura precária e assistemática de manutenção de arquivos dos serviços de saúde da cidade, temos os percentuais dos não localizados ou falsos positivos sem informação. Inexplicavelmente as DOs que tinham como causa básica ca de bexiga tiveram um percentual de não localizados acentuadamente elevado. Para o total de neoplasmas selecionados tivemos $12 \%$ e para outras causas $23 \%$.

Passando agora a apresentar os resultados mais significativos deste estudo de validação, que é diferente metologicamente falando da maioria dos estudos dessa natureza, e que possibilitou quantificar os FNs específicos por localização temos: Quatro FNs para os neoplasmas de localização não es- pecificada, 1 FN para leucemia, 3 para estômago, 3 para pulmāo, 2 para fígado e 1 para linfoma (Tabelas 1,2,3). Entre estes achados, temos que nenhum FN foi encontrado para ca de pâncreas e bexiga entre as mortes por outras causas, indicando todos os óbitos por estes neoplasmas foram representados pela DO. Entre esses óbitos, pode até ter alguns que não tenham sido causados exatamente por ca de pâncreas e de bexiga e sim por um neoplasma de outra localização. No entanto, todos os óbitos por estes neoplasmas foram efetivamente representados pela $\mathrm{DO}$.

Numa amostra de 481 DOs com causas não selecionadas foi encontrado um caso de leucemia, confirmado por mielograma, entre DOs que nem se re-

\section{TABELA 1}

Número e percentual de declarações de óbitos selecionadas segundo verdadeiros positivos, falsos positivos sem informação e número de falsos negativos encontrados entre outras causas de morte - Salvador, 1983.

\begin{tabular}{|c|c|c|c|c|c|c|c|c|}
\hline \multirow{2}{*}{$\begin{array}{l}\text { Localização } \\
\text { Anatômica }\end{array}$} & \multirow[b]{2}{*}{ Selecionados } & \multicolumn{2}{|c|}{ VP } & \multicolumn{2}{|c|}{$F P$} & \multicolumn{2}{|c|}{ FP-SI } & \multirow{2}{*}{$\begin{array}{r}\text { FN-OC } \\
N\end{array}$} \\
\hline & & $\mathrm{N}$ & $\%$ & $N$ & $\%$ & $N$ & $\%$ & \\
\hline Leucemia & 48 & 43 & 89,58 & 1 & 2,08 & 4 & 8,33 & 1 \\
\hline Pulmão & 87 & 70 & 80,45 & 10 & 11,49 & 7 & 8,04 & 3 \\
\hline Estômago & 91 & 65 & 71,42 & 13 & 14,28 & 13 & 14,28 & 3 \\
\hline Fígado & 41 & 25 & 60,97 & 10 & 24,39 & 6 & 44,63 & 2 \\
\hline Pâncreas & 31 & 14 & 45,16 & 12 & 38,70 & 5 & 16,12 & 0 \\
\hline Bexiga & 15 & 8 & 53,33 & 3 & 20,00 & 4 & 26,66 & 0 \\
\hline Linfomas & 53 & 43 & 81,13 & 5 & 9,43 & 5 & 9,43 & 1 \\
\hline Não especificado & 119 & 51 & 42,85 & 53 & 44,53 & 15 & 12,60 & 4 \\
\hline Total selecionados & 485 & 319 & 65,77 & 107 & 22,06 & 59 & 12,16 & 14 \\
\hline Outras causas & 481 & 249 & 51,76 & 121 & 25,15 & 111 & 23,07 & 0 \\
\hline
\end{tabular}

VP = Verdadeiro Posítivo;

$\mathrm{FP}=$ Falso positivo;

FP-SI: Falsos Positivos Sem Informação;

FN-OC: Falso negativo

\section{TABELA 2}

Distribuição de falsos negativos segundo causas registradas na declaração de óbito para as oito localizações anatômicas de câncer selecionadas — Salvador, 1983.

\begin{tabular}{|c|c|c|c|c|c|c|c|c|c|c|c|}
\hline \multicolumn{12}{|c|}{ Causas de origem } \\
\hline \multicolumn{2}{|c|}{$\begin{array}{l}\text { Localização Leucemia } \\
\text { Anatômica }\end{array}$} & \multirow{2}{*}{$\begin{array}{r}\text { Pulmäo } \\
0\end{array}$} & \multirow{2}{*}{$\begin{array}{c}\text { Estômago } \\
0\end{array}$} & \multirow{2}{*}{$\begin{array}{r}\text { Fígado } \\
0\end{array}$} & \multirow{2}{*}{$\begin{array}{r}\text { Pâncreas } \\
0\end{array}$} & \multirow{2}{*}{$\begin{array}{r}\text { Bexiga } \\
0\end{array}$} & \multirow{2}{*}{$\begin{array}{r}\text { Linfomas } \\
0\end{array}$} & \multirow{2}{*}{$\begin{array}{l}\begin{array}{l}\text { Não } \\
\text { Especif. }\end{array} \\
1\end{array}$} & \multirow{2}{*}{$\begin{array}{c}\text { Outras } \\
\text { Localiz. } \\
0\end{array}$} & \multirow{2}{*}{$\begin{array}{r}\text { Outras } \\
\text { Causas } \\
1\end{array}$} & \multirow{2}{*}{$\begin{array}{r}\text { Total } \\
2\end{array}$} \\
\hline Leucemia & 0 & & & & & & & & & & \\
\hline Pulmäo & 0 & 0 & 0 & 1 & 0 & 0 & 0 & 5 & 0 & 3 & 9 \\
\hline Estômago & 0 & 0 & 0 & 0 & 0 & 0 & 0 & 5 & 0 & 3 & 8 \\
\hline Fígado & 0 & 0 & 0 & 0 & 1 & 0 & 0 & 1 & 0 & 2 & 4 \\
\hline Pâncreas & 0 & 0 & 0 & 0 & 0 & 0 & 0 & 0 & 0 & 0 & 0 \\
\hline Bexiga & 0 & 0 & 0 & 0 & 0 & 0 & 0 & 4 & 0 & 0 & 4 \\
\hline Linformas & 0 & 0 & 1 & 1 & 3 & 0 & 0 & 1 & 0 & 1 & 7 \\
\hline \multicolumn{12}{|l|}{ Não } \\
\hline Especificado & 0 & 0 & 0 & 0 & 0 & 0 & 0 & 0 & 0 & 4 & 4 \\
\hline \multirow{2}{*}{\multicolumn{12}{|c|}{ Total Neoplasmas }} \\
\hline & as $\quad 0$ & 0 & 1 & 2 & 4 & 0 & 0 & 17 & 0 & 14 & 38 \\
\hline
\end{tabular}


feriam a câncer. Entre as 10.098 restantes então, serão provavelmente encontradas cerca de 20 outras mortes por leucemia. Note que as 966 DOs analisadas por este estudo já estão excluídas desse total.

Seguindo este raciocínio, com o número de FNs específicos por localização encontrados no "pool" de mortes por outras causas, chegamos ao percentual de subestimados.

É possível que $0,82 \%$ desse total seja na realidade óbitos por ca de localização não especificada (Tabela 4). Os cânceres de pulmão e estômago com percentuais de subestimados iguais, de 0,61 e fígado com $0,40 \%$. Para o total de neoplasmas selecionados o percentual de subestimados é de $2,9 \%$ enquanto, para os de outras localizações, é de $4,36 \%$.

Isto em valores absolutos é o mesmo que dizer que o número de mortes a mais variaria com $95 \%$ de certeza entre no mínimo 46 e no máximo 76 para os cânceres de pulmão e estômago. Esta variação seria de, no mínimo, 261 e, no máximo, 325 para os neoplasmas selecionados, enquanto que para as demais localizaçōes a variação é de no mínimo 400 e no máximo 479.

\section{DISCUSSÃO}

Este estudo analisou a precisão da causa básica de morte, enquanto fonte de informação, registrada em DOs de Salvador para o ano de 1983. Não há razão para acreditar que, para os anos anteriores a, e imediatamente posteriores a 1983, tenha ocorrido alguma mudança na maneira como os médicos preenchem as DOs. Portanto, os resultados desta pesquisa podem perfeitamente refletir a realidade dos anos adjacentes.

Os resultados revelaram de forma veemente que os percentuais de confirmação aqui encontrados acumulam maior quantidade de erro dificultando

\section{TABELA 3}

Distribuição dos falsos negativos por câncer segundo causas registradas na declaração de óbito — Salvador, 1983.

\begin{tabular}{|c|c|c|c|c|c|c|}
\hline \multicolumn{7}{|c|}{ Causas registradas na DO } \\
\hline $\begin{array}{l}\text { Localização } \\
\text { Anatômica }\end{array}$ & Estômago & Fígado & Pâncreas & $\begin{array}{c}\mathrm{Nāo} \\
\text { Especificado }\end{array}$ & $\begin{array}{l}\text { Outras } \\
\text { Causas }\end{array}$ & Total \\
\hline $\begin{array}{l}\text { Neoplasmas } \\
\text { Selecionados }\end{array}$ & 1 & 2 & 4 & 17 & 14 & 38 \\
\hline $\begin{array}{l}\text { Outras } \\
\text { Localizações }\end{array}$ & 0 & 0 & 0 & 23 & 21 & 44 \\
\hline Total & 1 & 2 & 4 & 40 & 35 & 82 \\
\hline
\end{tabular}

\section{TABELA 4}

Número oficial de mortes, número e percentual de mortes subestimadas, erro padräo do percentual, e $95 \%$ intervalo de confiança do número de mortes subestimadas por câncer — Salvador, 1983.

\begin{tabular}{|c|c|c|c|c|c|c|c|}
\hline \multirow{2}{*}{$\begin{array}{l}\text { Localização } \\
\text { Anatômica }\end{array}$} & \multirow{2}{*}{$\begin{array}{l}\text { Estatística } \\
\text { do } \\
\text { Ministęrio } \\
\text { Saúde }\end{array}$} & \multicolumn{2}{|c|}{ Mortes Subestimadas } & Erro Padrão & \multicolumn{3}{|c|}{ Mortes Subestimadas } \\
\hline & & $N$ & $\%$ & DO $\%$ & $95 \%$ & & IC \\
\hline Leucemia & 53 & 19 & 0,19 & 0,0008 & 10 & - & 27 \\
\hline Pulmão & 88 & 61 & 0,61 & 0,0015 & 46 & - & 76 \\
\hline Estômago & 91 & 61 & 0,61 & 0,0015 & 46 & - & 76 \\
\hline Fígado & 43 & 40 & 0,40 & 0,0012 & 28 & - & 52 \\
\hline Pâncreas & 33 & 0 & 0 & 0 & & 0 & \\
\hline Bexiga & 15 & 0 & 0 & 0 & & 0 & \\
\hline Linfomas & 53 & 19 & 0,19 & 0,0008 & 10 & - & 27 \\
\hline Nāo especificado & 132 & 82 & 0,82 & 0,0017 & 65 & - & 99 \\
\hline Total selecionados & 508 & 292 & 2,90 & 0,0032 & 261 & - & 325 \\
\hline Outras localizaçōes & 452 & 440 & 4,36 & 0,0039 & 400 & - & 479 \\
\hline
\end{tabular}


enormemente a interpretação dos mesmos. É possível que muitas mortes por câncer não tenham sido confirmadas não porque os pacientes não passaram pelos serviços ou pelos laboratórios de anatomia patológica mas, sim, porque os sistemas de arquivos dos serviços são organizados de forma precária e ineficiente. Todos que não foram localizados em hospitais e/ou laboratórios foram considerados como FP-SI. Temos ainda influenciando os percentuais de confirmação, erros no processo de codificação, erros provocados pelo preenchimento incompleto da DO, a questão do acesso aos serviços de saúde e, a ausência de testes diagnósticos satisfatoriamente sensiveis e específicos para o diagnóstico de alguns neoplasmas. Esta conjugação de fatores forçam os resultados na direção de deflação no que se refere à proporção de confirmados, todos eles, associados ao nosso estágio de subdesenvolvimento.

A proporção de subestimados, por outro lado, sofre menos interferências e não está tão sujeita a tendenciosidades como as aqui citadas, relacionadas com os percentuais de confirmados. Daí acreditarmos que, a DO reflete, difusamente, o número de mortes por câncer em Salvador, a um nível que quando erros sistemáticos estão operando, estes tendem a favorecer a subnotificação do câncer, dado que a proporçāo de subestimados está associada basicamente a:

1. questão do preenchimento inexato e irregular da DO pelos médicos;

2. situações raras que efetivamente eliminam a possibilidade de uma neoplasia ser referida como causa básica de morte. São elas: um caso de uma completa cura do câncer em questão, os casos de morte por causas externas tipo acidentes e suicídios, os casos de morte por uma outra patologia com o potencial de letalidade ainda maior que uma neoplasia de estômago, por exemplo.

Conclui-se portanto, que o número de mortes por câncer está subestimado quando as estatísticas de mortalidade são usadas como fonte de informação. A mortalidade proporcional sofrerá mudanças significativas para câncer de uma maneira geral e, em particular, para os cânceres de estốmago, pulmão e fígado. Estes neoplasmas foram significativamente subestimados. Por exemplo, se todos os indivíduos que foram a óbito com ca de estômago tivessem como causa básica este neoplasma, o número de mortes por ca de estômago cresceria de 91 para aproximadamente 152 , um aumento de $67 \%$. Cálculos similares poderiam ser aplicados para ca de pulmão e fígado causando mudanças de 69 e $93 \%$, respectivamente (Tabela 4 ).

Ficou mostrado também que as estatísticas de mortalidade para os neoplasmas selecionados estão menos sujeitas a erros de classificação que as existentes para os neoplasmas de localização anatômica não privilegiados por este estudo. Os neoplasmas selecionados estão subestimados em apenas $57 \%$ se incluirmos os de localização não especificada. Excluindo-os, este percentual decresce para $41 \%$ enquanto que para as demais localizações anatômicas o percentual de subestimados sobe, assustadoramente, para $96 \%$.

\section{AGRADECIMENTOS}

Aos hospitais e laboratórios e aos coletadores de dados que muito colaboraram para que esta pesquisa se realizasse.

SCHNITMAN, A. [Analysis of the declaration of the basic cause of death by cancer in Salvador, Brazil]. Rev. Saúde públ., S.Paulo, 24:490-6, 1990.

\begin{abstract}
The accuracy of the cancer mortality figures was determined by comparing the underlying causes of death as coded on death certificates with pathology reports and hospital diagnoses of a sample of 966 deaths of the total deaths occurring in Salvador during 1983. The death certificates were found to be accurate in $65 \%$ of 485 cancer deaths studied. Thirty-five histologically confirmed cancer deaths were found in a random sample of 481 deaths from other causes (460 stating other causes and 21 stating cancer sites that were not under study). This means that, approximately 700 more cancer deaths may be hidden among the remaining 10,098 death certificates.
\end{abstract}

KEYWORDS: Death certificates. Cause of death. Neoplasms, mortality.

\title{
REFERENCIAS BIBLIOGRÁFICAS
}

1. BARCLAY, T. H. C. et al. The accuracy of cancer diagnosis on death certificates. Cancer, 15: 5-9, 1962.

2. BURBANK, F. Patterns in cancer mortality in the United States: 1950-1967. Bethesda, Md, National Can- cer Institute, 1971. (Monograph, 33).

3. DORN, H. F, \& HORN, J. L. The reliability of certificates of deaths from cancer. Amer. J. Hyg., 34: 1223, 1941 . 
4. DORN, H. F. \& CUTTLER, S. J. Morbidity from cancer in United States: comparison of death certificates and case reports. Washington, D.C., Public Health Services. 1958. p. 117-24, 206-7.(Public Health Monographs, 56).

5. GITTELSOHN, A. et al. Studies on the reliability of vital and death records: comparison of cause of death and hospital record diagnosis. Amer. J. publ. Hlth, 69: 680-9, 1979.

6. KLEBBA, A. J. Comparability of mortality statistics: of the seventh and eighth revisions of the Intemational Classification of Diseases. Vital Hith Statist. Ser. 2, (66) 1975.

7. LAURENTI, R. \& MELLO JORGE, M. H. P. de $O$ atestado de b́bito. São Paulo, Centro da OMS para Classificação de Doenças em Português, 1981.

8. MINISTÉRIO DA SAÚDE. Secretaria Nacional de Açōes Básicas de Saúde. Divisão Nacional de Epidemio- logia. Estatística de mortalidade: 1980. Brasília, Centro de Documentação do Ministério da Saúde, 1983.

9. MORIYAMA, I. M. Inquiry into diagnostic evidence supporting medical certification of death. Amer. $J$. pul. Hlth, 48: 1376-87, 1958.

10. PERCY, C. et al. Accuracy of cancer death certificates and its effect on cancer mortality statistics. Amer. J. publ. Hlth, 71: 242-50, 1981.

11. STEER, A. et al. Accuracy of diagnosis of cancer among autopsy cases: JNIH-ABCC Population for Hiroshima and Nagasaki. Gann, Tokyo, 67: 625-32, 1976.

12. WORLD HEALTH ORGANIZATION. International classification of disease; 1975, revision. Geneva, 1977.

Recebido para publicação em 29/11/1989

Reapresentado em 12/7/1990 Aprovado para publicação em 14/8/1990 